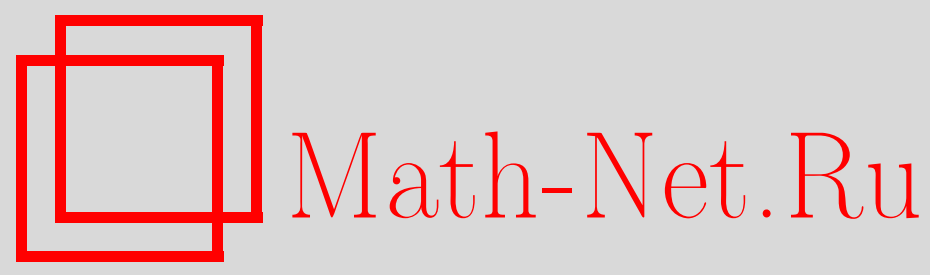

К. И. Осколков, Об одном результате Теляковского и кратных преобразованиях Гильберта с полиномиальными фазами, Матем. заметки, 2003, том 74, выпуск 2, 242-256

DOI: https://doi.org/10.4213/mzm261

Использование Общероссийского математического портала Math-Net.Ru подразумевает, что вы прочитали и согласны с пользовательским соглашением http://www.mathnet.ru/rus/agreement

Параметры загрузки:

IP : 35.173 .137 .237

26 апреля 2023 г., 13:34:53

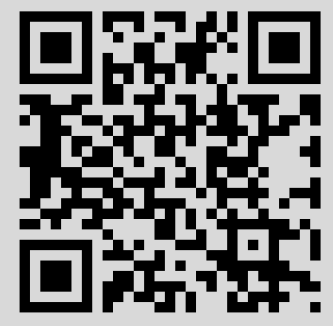




\section{ОБ ОДНОМ РЕЗУЛЬТАТЕ ТЕЛЯКОВСКОГО И КРАТНЫХ ПРЕОБРАЗОВАНИЯХ ГИЛЬБЕРТА С ПОЛИНОМИАЛЬНЫМИ ФАЗАМИ}

\section{К. И. Осколков}

В работе получен кратный аналог теоремы, доказанной в 1987 г. Г.И. Архиповым и автором, дающей оценку дискретных преобразований Гильберта с полиномиальной фазой. Для линейного случая соответствующая оценка суммы кратного тригонометрического ряда была установлена С. А. Теляковским.

Библиографиял: 17 названий.

Я посвящаю эту работу моему учителю Сергею Александровичу Теляковскому к его семидесятилетию.

Пусть $\mathbb{R}^{d}, d=1,2, \ldots,-d$-мерное вещественное евклидово пространство векторов $\mathbf{v}=\left(v_{1}, \ldots, v_{d}\right) ; \mathbb{Z}^{d}$-целочисленная решетка в $\mathbb{R}^{d} ; \mathbb{N}^{d}$-подмножество векторов $\mathbf{n} \in \mathbb{R}^{d}$ с натуральными координатами $n_{k} ; \mathbb{Z}^{d} / \mathbb{N}$ - множество векторов в $\mathbb{R}^{d}$ с рациональньми координатами.

Будем говорить, что подмножество $\omega \subset \mathbb{R}^{d}$ координатно выпуклое, если пересечение $\omega$ с любой прямой, параллельной какой-либо из координатных осей, является либо интервалом, либо пустым множеством. Обозначим через $\Omega^{d}$ класс всех координатно выпуклых множеств в $\mathbb{R}^{d}$.

Теляковский [1], [2] установил следующий замечательный результат о кратных sinрядах с линейной фазой.

Теорема 1. Для $d \geqslant 2$ выполнено

$$
\sup _{\omega \in \Omega^{d}} \sup _{\mathbf{x} \in \mathbb{R}^{d}}\left|\sum_{\mathbf{n} \in \omega \cap \mathbb{N}^{d}} \frac{\sin n_{1} x_{1}}{n_{1}} \ldots \frac{\sin n_{d} x_{d}}{n_{d}}\right|<\infty .
$$

Эта теорема нашла многочисленные применения, в частности, в проблеме оценок поперечников по Колмогорову классов функций с ограниченной смешанной производной и, в целом, в теме приближения гиперболическим крестом, а также в теории сходимости кратньх рядов Фурье функций многих переменных, имеющих ограниченную вариацию в смысле Харди, см. [3].

Следуюший результат был установлен в [4] на основе метода тригонометрических сумм Виноградова [5]. Он касается одномерных дискретных преобразований Гильберта с полиномиальной фазой высшей степени (см. также [6]). Обозначение $\mathscr{P}^{r}, r \in \mathbb{N}$, используется для множества алгебраических многочленов $p$ одной переменной с вещественньпи коэффициентами степени $r$ таких, что $p(0)=0$.

Работа выполнена при поддержке NSF, грант DMS-9706883. 
Теорема 2. Для многочлена $p \in \mathscr{P}^{r}$ положим

$$
h_{m}(p):=\sum_{n=1}^{m} \frac{e^{i p(n)}-e^{i p(-n)}}{n}, \quad m \geqslant 1 .
$$

Tогда

$$
\sup _{p \in \mathscr{P}^{\circ} r} \sup _{m}\left|h_{m}(p)\right|<\infty
$$

и для каждого фиксированного многочлена $p \in \mathscr{P}^{r}$ существует предел

$$
h(p):=\lim _{m \rightarrow \infty} h_{m}(p) .
$$

Этот результат также нашел разнообразные приложения. Они включают решение некоторых задач о спектрах равномерной сходимости и исследование свойств решений задачи Коши для широкого класса линейных дифференциальных уравнений в частных производных типа Шрёдингера с периодическими начальными данными. По сути, теорема 2 стала отправной точкой для изучения широкого класса рядов Виноградова

$$
V\left(f ; x_{1}, \ldots, x_{r}\right):=\sum_{n \in \mathbb{Z}} c_{n}(f) e^{2 \pi i\left(x_{1} n+x_{2} n^{2}+\cdots+x_{r} n^{r}\right)}, \quad c_{n}(f):=\int_{0}^{1} f(x) e^{-2 \pi i n x} d x .
$$

Обзор некоторых результатов в этих направлениях содержится в работе [7], а дальнейшие развития - в [8], [9].

Здесь будет доказана теорема 3 о кратных дискретных преобразованиях Гильберта с полиномиальными фазами. Она обобщает и несколько усиливает теоремы 1 и 2.

Будем говорить, что числовая последовательность $f=\left\{f_{n}\right\}_{n \in \mathbb{N}}$ медленная, если она удовлетворяет условию Литтлвуда-Пэли (см. [10, гл. 15]):

$$
\|f\|_{\mathscr{S}}:=\|f\|_{\infty}+\sup _{n} \sum_{n \leqslant m \leqslant 2 n}\left|f_{m}-f_{m+1}\right|<\infty, \quad\|f\|_{\infty}:=\sup _{n}\left|f_{n}\right| .
$$

Обозначим через $\mathscr{S}$ класс всех последовательностей с условием $\|f\|_{\mathscr{S}}<\infty$. Ясно, что если последовательность $f$ имеет ограниченную вариацию в обычном смысле, т.е. $f \in \mathrm{BV}$, то эта последовательность медленная. Однако класс $\mathscr{S}$ существенно шире, чем BV. Например, очевидно, что для любого фиксированного вещественного $t \neq 0$ мы имеем $f_{t}:=\left\{n^{i t}\right\}=\left\{e^{i t \ln n}\right\}_{n \in \mathbb{N}} \in \mathscr{S}, f_{t} \notin \mathrm{BV}$.

Мы будем говорить, что $d$-индексная последовательность $f=\left\{f_{\mathbf{n}}\right\}_{\mathbf{n} \in \mathbb{N} d}$ координатно медленная (обозначение: $f \in \mathscr{S}^{d}$ ), если сужения $f$ на прямые, параллельные координатным осям, являются равномерно медленными:

$$
\|f\|_{\mathscr{S} d}:=\|f\|_{\infty}+\max _{1 \leqslant k \leqslant d} \sup _{\mathbf{n}_{k}} \sup _{n} \sum_{n \leqslant m \leqslant 2 n}\left|f_{\mathbf{n}_{k}+m \mathbf{e}_{k}}-f_{\mathbf{n}_{k}+(m+1) \mathbf{e}_{k}}\right|<\infty,
$$

где

$$
\mathbf{e}_{k}:=(0, \ldots, 0,1,0, \ldots, 0), \quad k=1, \ldots, d,
$$

обозначает стандартньй базис в $\mathbb{R}^{d}$ и для $\mathbf{n}=\left(n_{1}, \ldots, n_{k}, \ldots, n_{d}\right)$ используется обозначение $\mathbf{n}_{k}:=\mathbf{n}-n_{k} \mathbf{e}_{k}$. Очевидно, что характеристическая функция координатно выпуклой области, т.е. $\omega \in \Omega^{d}$, является координатно медленной.

Будут также использоваться следующие обозначения: $\mathscr{P} r, d-$ множество наборов многочленов $\vec{p}=\left(p_{1}, \ldots, p_{d}\right)$, где $p_{k} \in \mathscr{P}^{r} ; \square_{\mathbf{m}}-$ параллелепипед $\left\{\mathbf{n} \in \mathbb{N}^{d}: n_{k} \leqslant m_{k}\right.$, $k=1, \ldots, d\}$. Символы $\stackrel{r}{\ll}, \stackrel{r, d}{\ll}$ в соотношениях типа $A \stackrel{r}{\ll} B, A \stackrel{r, d}{\ll} B$ обозначают, что существуют конечные множители $c_{r}, c_{r, d}$, которые зависят только от указанных параметров $(r$ или $r, d)$ такие, что $|A| \leqslant c_{r}|B|$ или соответственно $|A| \leqslant c_{r, d}|B|$. 
ТЕОРема 3. Для $\vec{p} \in \mathscr{P}^{r, d}, \mathbf{m} \in \mathbb{N}^{d}$ положим

$$
h_{\mathbf{m}}(f, \vec{p}):=\sum_{\mathbf{n} \in \square_{\mathbf{m}}} f_{\mathbf{n}} \prod_{k=1}^{d} \frac{e^{i p_{k}\left(n_{k}\right)}-e^{i p_{k}\left(-n_{k}\right)}}{n_{k}} .
$$

Ecлu $f \in \mathscr{S}^{d}$, mo

$$
\sup _{\mathbf{m} \in \mathbb{N}^{d}} \sup _{\vec{p}}\left|h_{\mathbf{m}}(f, \vec{p})\right| \stackrel{r, d}{\ll}\|f\|_{\mathscr{S}^{d}}
$$

и для любого набора многочленов $\vec{p} \in \mathscr{P} r, d$ предел $h(f, \vec{p}):=\lim _{\min _{k}} m_{k} \rightarrow \infty h_{\mathbf{m}}(f, \vec{p})$ существует.

Интересно сравнить эту теорему со следующим результатом отрицательного характера, полученным недавно Гараевым [11]: существует такое действительное число $x$, что последовательность

$$
h_{N}:=\sum_{m=1}^{N} \sum_{n=1}^{N} \frac{\sin (m n x)}{m n}
$$

расходится при $N \rightarrow \infty$.

ДоКАЗАТЕЛЬСТВО ТЕОРЕМЫ 3 . Для $p \in \mathscr{P}^{r}$ положим

$$
e_{-}^{i p(t)}:=\frac{e^{i p(t)}-e^{i p(-t)}}{2 i}
$$

и для $n \in \mathbb{N}, \mathbf{n} \in \mathbb{N}^{d}, \vec{p} \in \mathscr{P}^{r, d}$ рассмотрим следуюшие тригонометрические суммы и интегралы:

$$
\begin{gathered}
T_{n}(p):=\frac{1}{n} \sum_{m=1}^{n} e_{-}^{i p(n)}, \quad I_{n}(p):=\frac{1}{n} \int_{0}^{n} e_{-}^{i p(t)} d t, \quad T_{0}(p)=I_{0}(p):=0 \\
A_{n}(p):=T_{n}(p)-T_{n-1}(p), \quad B_{n}(p):=B_{n}(p)-B_{n-1}(p), \\
A_{\mathbf{n}}(\vec{p}):=\prod_{k=1}^{d} A_{n_{k}}\left(p_{k}\right), \quad B_{\mathbf{n}}(\vec{p}):=\prod_{k=1}^{d} B_{n_{k}}\left(p_{k}\right) .
\end{gathered}
$$

Имеем

$$
\frac{e_{-}^{i p(n)}}{n}=A_{n}(p)+\frac{T_{n-1}(p)}{n}, \quad \int_{n-1}^{n} \frac{e_{-}^{i p(t)}}{t} d t=B_{n}(p)+\frac{I_{n-1}(p)}{n} .
$$

В [4] установлено, что

$$
\text { a) } \sup _{p \in \mathscr{P}^{r}} \sum_{n \in \mathbb{N}} \frac{\left|T_{n-1}(p)\right|}{n}<\infty, \quad \text { b) } \sup _{p \in \mathscr{P}^{r}} \sum_{n \in \mathbb{N}} \frac{\left|I_{n-1}(p)\right|}{n}<\infty \text {. }
$$

Утверждение b) относится к интегральным преобразованиям Гильберта

$$
g(p):=\lim _{m \rightarrow \infty} g_{m}(p), \quad g_{m}(p):=\int_{0}^{m} \frac{e_{-}^{i p(t)}}{t} d t
$$


Равномерная ограниченность $\sup _{m>0} \sup _{p \in \mathscr{P} r}\left|g_{m}(p)\right|<\infty$ и существование предела $g(p)$ - результаты, установленные Стейном и Вейнгером [12].

Утверждение а) в (4) более сложное, потому что существенную роль играют арифметические характеристики вектора коэффициентов многочлена $p$ и доказательство требует применения кругового метода Харди-Литтлвуда-Виноградова. Это утверждение было установлено в работе [4]. Независимо и несколько позже, чем в [4], глобальная ограниченность $\sup _{m>0} \sup _{p \in \mathscr{P}^{r}}\left|h_{m}(p)\right|<\infty$, см. (1), была доказана Стейном и Вейнгером, см. [6], а также [13, с. 373].

Для подмножества индексов $\mathscr{A} \subset[1, d]$ (возможно пустого) рассмотрим составные интегрально-дискретные произведения

$$
C_{\mathbf{n}}(\mathscr{A}, \vec{p}):=\left(\prod_{k \in \mathscr{A}} A_{n_{k}}\left(p_{k}\right)\right)\left(\prod_{k \in[1, d] \backslash \mathscr{A}} B_{n_{k}}\left(p_{k}\right)\right), \quad \mathbf{n} \in \mathbb{N}^{d}, \quad \vec{p} \in \mathscr{P}^{r, d}
$$

Тогда ввиду (3) и (4) теорема 3 есть следствие, соответствующее частному случаю $\mathscr{A}=[1, d]$, из следующего утверждения.

Лемма 1. Допустим, что $f:=\left\{f_{\mathbf{n}}\right\}_{\mathbf{n} \in \mathbb{N}^{d}}-$ координатно медленная последовательность $,\|f\|_{\mathscr{S} d} \leqslant 1, \mathscr{A} \subset[1, d]-$ подмножество индексов и $\vec{p} \in \mathscr{P}^{r, d}$. Тогда предел

$$
S(f, \mathscr{A}, \vec{p}):=\lim _{\min _{k}} \lim _{m_{k} \rightarrow \infty} \sum_{\mathbf{n} \in \square_{m}} f_{\mathbf{n}} C_{\mathbf{n}}(\mathscr{A}, \vec{p})
$$

существует и

$$
|S(f, \mathscr{A}, \vec{p})| \stackrel{r, d}{\ll} 1 .
$$

ДокАЗАТЕльСтво. Для многочлена $p \in \mathscr{P}^{r}$ обозначим через $\mathbf{x}=\left(x_{1}, \ldots, x_{r}\right)$ вектор коэффициентов многочлена $p /(2 \pi)$, и пусть

$$
p(t)=2 \pi P(\mathbf{x}, t):=2 \pi\left(x_{1} t+\cdots+x_{r} t^{r}\right), \quad p_{*}(t):=2 \pi\left(\left|x_{1}\right| t+\cdots+\left|x_{r}\right| t^{r}\right) .
$$

Следуюшие оценки тривиальны:

$$
\begin{gathered}
\max \left(\left|T_{n}(p)\right|,\left|I_{n}(p)\right|\right) \leqslant \min \left(1, p_{*}(n)\right), \\
\left|C_{n}(p)\right| \leqslant \max \left(\left|A_{n}(p)\right|,\left|B_{n}(p)\right|\right) \ll \frac{\min \left(1, p_{*}(n)\right)}{n}
\end{gathered}
$$

просто в силу того, что $\left|e_{-}^{i p}\right|=\left|\sin p_{-}\right|$, где $p_{-}(t):=(p(t)-p(-t)) / 2$, и $\left|p_{-}\right| \leqslant p_{*}$.

Мы разобьем доказательство леммы 1 на 4 следуюших случая:

1) $\mathscr{A}=\varnothing, d=1$

2) $\mathscr{A}=\varnothing, d \geqslant 2$

3) $\mathscr{A}=\{1\}, d=1$;

4) общее множество $\mathscr{A} \subset[1, d], d \geqslant 2$. 
Случай 1) самый простой. Здесь нужно рассмотреть сумму интегралов

$$
S(f, p)=S(f, \varnothing, p):=\sum_{n \in \mathbb{N}} f_{n} B_{n}(p) .
$$

Применяя преобразование Абеля для $m$-ой частной суммы, имеем

$$
\begin{aligned}
S_{m}(f, p) & :=\sum_{n=1}^{m} f_{n} B_{n}(p)=\sum_{n=1}^{m} f_{n}\left(I_{n}(p)-I_{n-1}(p)\right) \\
& =f_{m} I_{m}(p)+\sum_{n=1}^{m-1} I_{n}(p) \Delta f_{n}, \quad \Delta f_{n}:=f_{n}-f_{n+1} .
\end{aligned}
$$

Поэтому, достаточно доказать, что $I_{n}(p) \rightarrow 0, n \rightarrow \infty$ и если $f \in \mathscr{S},\|f\|_{\mathscr{S}} \leqslant 1$, то

$$
\sum_{n=1}^{\infty}\left|I_{n}(p) \Delta f_{n}\right| \stackrel{r}{\ll} 1
$$

Из определения медленной последовательности вытекает, что

$$
\sum_{n=1}^{m} p_{*}(n)\left|\Delta f_{n}\right| \ll p_{*}(m), \quad \sum_{n=m+1}^{\infty} p_{*}^{-\rho}(n)\left|\Delta f_{n}\right| \stackrel{\rho}{\ll} p_{*}^{-\rho}(m), \quad p \in \mathscr{P}^{r}, \quad \rho>0 .
$$

Далее, для интеграла $I_{n}$ вьполнена следующая оценка (см. также [4], [8]):

$$
\left|I_{n}(p)\right| \ll \min \left(p_{*}(n), p_{*}^{-\rho}(n)\right), \quad \rho:=\frac{1}{r} .
$$

Ввиду (6) эта оценка является следствием неравенства

$$
\left|\frac{1}{n} \int_{0}^{n} e^{i p(t)} d t\right| \ll \min \left(1, p_{*}^{-\rho}(n)\right), \quad p \in \mathscr{P}^{r},
$$

которое эквивалентно известной оценке Виноградова стандартного осцилляционного интеграла с полиномиальной фазой (см. [5, гл. 2, лемма 4]):

$$
\left|\int_{0}^{1} e^{i p(t)} d t\right|=\left|\int_{0}^{1} e^{2 \pi i P(\mathbf{x}, t)} d t\right| \leqslant \min \left(1,32 \min _{k}\left|x_{k}\right|^{-\rho}\right) \leqslant \min \left(1,32 r^{\rho} p_{*}^{-\rho}(1)\right) .
$$

Поэтому из (9) вытекает, что, и в самом деле, $I_{n}(p) \rightarrow 0, n \rightarrow \infty$, и

$$
\sum_{n=1}^{\infty}\left|I_{n}(p) \Delta f_{n}\right| \ll \sum_{n=1}^{\infty} \min \left(p_{*}(n), p_{*}^{-\rho}(n)\right)\left|\Delta f_{n}\right| \stackrel{r}{\ll} \min _{m}\left(p_{*}(m)+p_{*}^{-\rho}(m)\right) \stackrel{r}{\ll} 1 .
$$

Более того, мы имеем

$$
\begin{aligned}
\left|S(f, p)-S_{m}(f, p)\right| & =\left|\sum_{n=m}^{\infty} f_{n} B_{n}(p)\right| \leqslant\left|f_{m} I_{m}(p)\right|+\sum_{n=m}^{\infty}\left|I_{n}(p) \Delta f_{n}\right| \\
& \ll \sum_{n=m}^{\infty} \min \left(p_{*}(n), p_{*}^{-\rho}(n)\right)\left|\Delta f_{n}\right| \stackrel{r}{\ll} \min \left(1, p_{*}^{-\rho}(m)\right),
\end{aligned}
$$


что представляет собой оценку скорости сходимости интеграла в случае 1) и завершает доказательство леммы 1 в случае 1$)$.

Случай 2). Здесь надо рассмотреть кратные суммы интегралов

$$
S(f, \vec{p})=S(f, \varnothing, \vec{p})=\sum_{\mathbf{n} \in \mathbb{N}^{d}} f_{\mathbf{n}} B_{\mathbf{n}}(\vec{p}) .
$$

Мы воспользуемся модификацией основной идеи Теляковского доказательства теоремы 1. Для данного набора многочленов $\vec{p}=\left(p_{1}, \ldots, p_{d}\right) \in \mathscr{P}^{r, d}$ разобьем область суммирования $\mathbb{N}^{d}$ на $d$ ! подобластей в соответствии с величиной многочленов $p_{* 1}, \ldots, p_{* d}$. Типичными подобластями являются “алгебраические октанты"

$$
\begin{aligned}
\omega=\omega(\vec{p}) & :=\left\{\left(n_{1}, \ldots, n_{d}\right) \in \mathbb{N}^{d}: p_{* 1}\left(n_{1}\right) \geqslant \cdots \geqslant p_{* d}\left(n_{d}\right)\right\} \\
\omega_{m}=\omega_{m}(\vec{p}) & :=\left\{\left(n_{1}, \ldots, n_{d}\right) \in \mathbb{N}^{d}: p_{* 1}\left(n_{1}\right) \geqslant \cdots \geqslant p_{* d}\left(n_{d}\right), n_{d} \geqslant m\right\}, \quad m \in \mathbb{N} .
\end{aligned}
$$

Другие октанты (их количество равно $d !-1$ ) получаются из $\omega$ всевозможными перестановками неравенств между многочленами $p_{* k}\left(n_{k}\right)$ и заменой $\leqslant$ на строгие неравенства < везде или в части неравенств, так что получающиеся подмножества не имеют общих точек и разбивают все $\mathbb{N}^{d}$.

Докажем, что для последовательности $f$ с компактным носителем и такой, что $\|f\|_{\mathscr{S} d} \leqslant 1$, выполнено

$$
\left|S_{\omega_{m}}\right| \stackrel{r, d}{\ll} \min \left(1, p_{*}-\rho(m)\right), \quad m \in \mathbb{N}, \quad S_{\omega_{m}}:=\sum_{\mathbf{n} \in \omega_{m}} f_{\mathbf{n}} B_{\mathbf{n}}(\vec{p}) .
$$

Для краткости положим $n_{1}:=n, p_{1}:=p$ и для $\mathbf{n}=\left(n, n_{2}, \ldots, n_{d}\right) \in \mathbb{N}^{d}, m \in \mathbb{N}$, $k=1, \ldots, d-1$ обозначим $\mathbf{n}^{k}:=\left(n_{k+1}, \ldots, n_{d}\right) \in \mathbb{N}^{d-k}$,

$$
\begin{gathered}
B_{\mathbf{n}^{k}}(\vec{p}):=\prod_{l=k+1}^{d} B_{n_{l}}\left(p_{l}\right), \quad \Pi_{\mathbf{n}^{k}}(\vec{p}):=\prod_{l=k+1}^{d} \frac{\min \left(1, p_{* l}\left(n_{l}\right)\right)}{n_{l}} \\
\omega_{m}^{k}:=\left\{\mathbf{n}^{k} \in \mathbb{N}^{d-k}: p_{* k+1}\left(n_{k+1}\right) \geqslant \cdots \geqslant p_{* d}\left(n_{d}\right), n_{d} \geqslant m\right\} .
\end{gathered}
$$

Тогда

$$
S_{\omega_{m}}=\sum_{\mathbf{n}^{1} \in \omega_{m}^{1}} B_{\mathbf{n}^{1}}(\vec{p})\left(\sum_{n: p_{*}(n) \geqslant p_{* 2}\left(n_{2}\right)} f_{\mathbf{n}} B_{n}(p)\right)
$$

и в силу $(10),(8)$

$$
\left|\sum_{n: p_{*}(n) \geqslant p_{* 2}\left(n_{2}\right)} f_{\mathbf{n}} B_{n}(p)\right| \stackrel{r}{\ll} \min \left(1, p_{* 2}^{-\rho}\left(n_{2}\right)\right), \quad\left|B_{\mathbf{n}^{1}}(\vec{p})\right| \stackrel{d}{\ll} \Pi_{\mathbf{n}^{1}}(\vec{p}) .
$$


Поэтому соотношение (12) вытекает из следующей цепочки неравенств:

$$
\begin{aligned}
\left|S_{\omega_{m}^{d}}\right| & \stackrel{r, d}{\ll} \sum_{\mathbf{n}^{1} \in \omega_{m}^{1}} \Pi_{\mathbf{n}^{1}}(\vec{p}) \min \left(1, p_{* 2}^{-\rho}\left(n_{2}\right)\right) \\
& =\sum_{\mathbf{n}^{2} \in \omega_{m}^{2}} \Pi_{\mathbf{n}^{2}}(\vec{p})\left(\sum_{n_{2}: p_{* 2}\left(n_{2}\right) \geqslant p_{* 3}\left(n_{3}\right)} \frac{\min \left(p_{* 2}\left(n_{2}\right), p_{* 2}-\rho\left(n_{2}\right)\right)}{n_{2}}\right) \\
& \stackrel{r}{\ll} \sum_{\mathbf{n}^{2} \in \omega_{m}^{2}} \Pi_{\mathbf{n}^{2}}(\vec{p}) \min \left(1, p_{* 3}^{-\rho}\left(n_{3}\right)\right) \stackrel{r, d}{\ll} \ldots \\
& \left.\stackrel{r, d}{\ll} \sum_{n_{d}=m}^{\infty} \frac{\min \left(p_{* d}\left(n_{d}\right), p_{*}-\rho\right.}{n_{d}}\left(n_{d}\right)\right)
\end{aligned}
$$

Ясно, что оценка (15) влечет глобальную ограниченность сумм $S(f, \vec{p})$ для произвольной координатно медленной последовательности $f,\|f\|_{\mathscr{S} d} \leqslant 1$, с компактньм носителем:

$$
\sup _{\vec{p} \in \mathscr{P} r, d}|S(f, \vec{p})| \stackrel{r, d}{\ll} 1 .
$$

Из (15) также вытекает сходимость бесконечного ряда в смысле Прингсхейма, если $f$ не имеет компактного носителя. Более того, справедлива следующая оценка для скорости сходимости последовательности прямоугольных частных сумм:

$$
\begin{gathered}
S_{\mathbf{m}}(f, \vec{p}):=\sum_{\mathbf{n} \in \square_{\mathbf{m}}} f_{\mathbf{n}} B_{\mathbf{n}}(\vec{p}), \quad \mu=\mu(\mathbf{m}):=\min _{k} m_{k}, \\
\left|S(f, \vec{p})-S_{\mathbf{m}}(f, \vec{p})\right| \stackrel{r, d}{\ll} \min \left(1, \max _{1 \leqslant k \leqslant d} p_{* k}^{-\rho}(\mu)\right) .
\end{gathered}
$$

Случай 3). Здесь $\mathscr{A}=\{1\}$, и нам надо рассмотреть простую дискретную тригонометрическую сумму

$$
S(f, p)=S(f,\{1\}, p):=\sum_{n \in \mathbb{N}} f_{n} A_{n}(p) .
$$

Как и выше, применяя преобразование Абеля, для частной суммы ряда имеем

$$
S_{m}(f, p):=\sum_{n=1}^{m} f_{n} A_{n}(p)=\sum_{n=1}^{m} f_{n}\left(T_{n}(p)-T_{n-1}(p)\right)=f_{m} T_{m}(p)+\sum_{n=1}^{m-1} T_{n}(p) \Delta f_{n}
$$

Поэтому, в силу теоремы 2 и оценки а) из (4) в этом случае нам надо доказать, что если $f$ - медленная последовательность, то

$$
\sup _{p \in \mathscr{P}^{r}} \sum_{n=1}^{\infty}\left|T_{n}(p) \Delta f_{n}\right|<\infty
$$

Хотя доказательство этого утверждения по сути такое же, как и доказательство оценки а) из (4) в статье [4], мы воспроизведем здесь наиболее существенные детали. 
Эти детали потребуются для полного доказательства нашей теоремы и, вероятно, будут полезны для читателя.

Дадим сначала общее описание конструкции. Для данного многочлена $p \in \mathscr{P}^{r}$ область суммирования $\mathbb{N}$ разбивается на два непересекаюшихся множества. Каждое из этих множеств является объединением попарно непересекающихся интервалов натуральных чисел:

$$
\begin{gathered}
\mathbb{N}=\mathbb{N}_{1}(p) \cup \mathbb{N}_{2}(p), \\
\mathbb{N}_{1}(p)=\bigcup_{j \geqslant 1}\left[\mu_{j}, \nu_{j}\right], \quad \mathbb{N}_{2}(p)=\bigcup_{j \geqslant 1}\left(\nu_{j}, \mu_{j+1}\right), \quad \mu_{j}=\mu_{j}(p), \quad \nu_{j}=\nu_{j}(p) .
\end{gathered}
$$

Это разбиение производится в соответствии с арифметическими свойствами вектора коэффициентов $\mathbf{x}$ многочлена $p /(2 \pi)=P(\mathbf{x}, \cdot)$. А именно, разбиение зависит от аппроксимации $\mathbf{x}$ векторами с рациональными координатами. Для $n \in\left[\mu_{j}, \nu_{j}\right]$ точка $\mathbf{x}$ находится вблизи к рациональной точке $\mathbf{y}_{j}$ c “относительно малым знаменателем". Здесь для тригонометрических сумм $T_{n}, A_{n}$ выполнены асимптотические формулы:

$$
\begin{gathered}
T_{n}(p)=\sigma_{j} I_{n}\left(\widetilde{p}_{j}\right)+\varepsilon_{n}, \quad A_{n}(p)=\sigma_{j} B_{n}\left(\widetilde{p}_{j}\right)+\left(\varepsilon_{n}-\varepsilon_{n-1}\right), \quad n \in\left[\mu_{j}, \nu_{j}\right], \\
\sigma_{j}=\sigma_{j}(\mathbf{x}), \quad \sum_{j}\left|\sigma_{j}\right| \stackrel{r}{\ll} 1, \quad \widetilde{p}_{j} \in \mathscr{P}^{r}, \quad \varepsilon_{n} \stackrel{r}{\ll} n^{-\alpha}, \quad \alpha=\alpha(r)>0 .
\end{gathered}
$$

Напротив, если $n \in \mathbb{N}_{2}(p)$, то $\mathbf{x}$ держится в стороне от рациональных векторов с мальми знаменателями. Для таких $n$ вьполнены оценки

$$
T_{n}(p)=\varepsilon_{n}, \quad A_{n}(p)=\varepsilon_{n}-\varepsilon_{n-1}, \quad\left|\varepsilon_{n}\right| \stackrel{r}{\ll} n^{-\beta}, \quad \beta=\beta(r)>0 .
$$

Допустим, что такая конструкция осуществима. Тогда мы оценим сумму (17), используя (19) и (20), следующим образом:

$$
\begin{aligned}
& \sum_{n \in\left[\mu_{j}, \nu_{j}\right]}\left|I_{n}\left(\widetilde{p}_{j}\right) \Delta f_{n}\right| \leqslant \sup _{\tilde{p} \in \mathscr{P} r} \sum_{n \in \mathbb{N}}\left|I_{n}(\widetilde{p}) \Delta f_{n}\right| \stackrel{r}{\ll} 1 ; \\
& \sum_{n}\left|T_{n}(p) \Delta f_{n}\right|=\sum_{n \in \mathbb{N}_{1}}\left|T_{n}(p) \Delta f_{n}\right|+\sum_{n \in \mathbb{N}_{2}}\left|T_{n}(p) \Delta f_{n}\right| \\
& \stackrel{r}{\ll} \sum_{j}\left|\sigma_{j}\right| \sum_{n \in\left[\mu_{j}, \nu_{j}\right]}\left|I_{n}\left(\widetilde{p}_{j}\right) \Delta f_{n}\right|+\sum_{n}\left(n^{-\alpha}+n^{-\beta}\right) \Delta f_{n} \\
& \stackrel{r, \alpha, \beta}{\ll} \sum_{j}\left|\sigma_{j}\right|+1 \stackrel{r, \alpha, \beta}{\ll} 1 .
\end{aligned}
$$

Для конкретной реализации такого построения мы воспользуемся круговьм методом Харди-Литтлвуда-Виноградова. Этот метод создан для получения асимптотических формул и оценок тригонометрических сумм Вейля

$$
E_{n}(p)=E_{n}(\mathbf{x}):=\frac{1}{n} \sum_{t=1}^{n} e^{i p(t)}=\frac{1}{n} \sum_{t=1}^{n} e^{2 \pi i P(\mathbf{x}, t)} .
$$


Если одна из координат $x_{1}, \ldots, x_{r}$ вектора $\mathbf{x}$ - иррациональное число, то согласно теореме Вейля [14]

$$
\lim _{n \rightarrow \infty} E_{n}(p)=0 \text {. }
$$

Для рациональной точки $\mathbf{y} \in \mathbb{Z}^{r} / \mathbb{N}$ обозначим $Q=Q(\mathbf{y})$ наименьшее общее кратное знаменателей координат в несократимом представлении и перепишем у в виде $\mathbf{y}=\mathbf{a} / Q$ :

$$
\begin{gathered}
\mathbf{y}=\left(\frac{b_{1}}{q_{1}}, \ldots, \frac{b_{r}}{q_{r}}\right) \in \mathbb{Z}^{r} / \mathbb{N}^{r}, \quad \mathbf{b} \in \mathbb{Z}^{r}, \quad \mathbf{q} \in \mathbb{N}^{r}, \quad\left(b_{s}, q_{s}\right)=1, \\
Q=Q(\mathbf{y}):=\left[q_{1}, \ldots, q_{r}\right], \quad \mathbf{y}=\left(\frac{a_{1}}{Q}, \ldots, \frac{a_{r}}{Q}\right)=\frac{\mathbf{a}}{Q}, \quad \mathbf{a} \in \mathbb{Z}^{r}, \quad Q \in \mathbb{N} .
\end{gathered}
$$

Для такого рационального у обозначим через $\sigma(\mathbf{y})$ соответствующую (нормированную) полную рациональную тригонометрическую сумму (сумму Гаусса высшего порядка), т.e.

$$
\sigma(\mathbf{y}):=\frac{1}{Q} \sum_{n=1}^{Q} e^{2 \pi i P(\mathbf{y}, n)}=\frac{1}{Q} \sum_{n=1}^{Q} e^{2 \pi i\left(a_{1} n+\cdots+a_{r} n^{r}\right) / Q}, \quad Q=Q(\mathbf{y}) .
$$

Имеем

$$
\lim _{n \rightarrow \infty} E_{n}(p)=E_{Q}(p)=\sigma(\mathbf{y}), \quad p=2 \pi P(\mathbf{y}, \cdot),
$$

и для $\sigma(\mathbf{y})$ вьполнены оценки Хуа Ло Гена (доказательство см. [15], [16])

$$
|\sigma(\mathbf{y})| \stackrel{r}{\ll} Q^{-\rho}(\mathbf{y}), \quad \rho=\frac{1}{r} .
$$

Как и в работе [4], мы воспользуемся результатами Архипова [17] в круговом методе. Соответственно, пространство $\mathbb{R}^{r}$ разбивается для данного натурального $n$ на два множества $\mathbb{R}^{r}=\mathscr{E}_{n} \cup \mathscr{F}_{n}$. Вектор коэффициентов $\mathbf{x} \in \mathbb{R}^{r}$ многочлена $p=P(\mathbf{x}, \cdot) /(2 \pi) \in \mathscr{P}^{r}$ принадлежит $\mathscr{E}_{n}$ (или большой дуге), если в малой прямоугольной окрестности $\mathbf{x}$ находится рациональная точка $\mathbf{y}=\left(y_{1}, \ldots, y_{r}\right)$ с малым знаменателем $Q(\mathbf{y})$ :

$$
\max _{1 \leqslant s \leqslant r} n^{s}\left|x_{s}-y_{s}\right|=\max _{1 \leqslant s \leqslant r} n^{s}\left|x_{s}-\frac{a_{s}}{Q}\right| \leqslant n^{0.3}, \quad Q=Q(\mathbf{y}) \leqslant n^{0.3} .
$$

Все $\mathbf{x} \in \mathbb{R}^{r}$, которые не обладают этим свойством, по определению составляют множество $\mathscr{F}_{n}$ (или малую дугу).

Необходимые элементы конструкции (18)-(20) содержатся в следующих утверждениях.

ЛЕмма 2 (Архипов [17, леммы 7 и 6]). 1) Если $\mathbf{x} \in \mathscr{E}_{n}$ и $\mathbf{y}-$ рациональная точка, удовлетворяющая (23), $\mathbf{z}:=\mathbf{x}-\mathbf{y}$, то

$$
E_{n}(p)=\sigma(\mathbf{y}) \frac{1}{n} \int_{0}^{n} e^{i \widetilde{p}(t)} d t+\varepsilon, \quad \widetilde{p}:=2 \pi P(\mathbf{z}, \cdot), \quad|\varepsilon| \leqslant 9 r Q n^{-1} \stackrel{r}{\ll} n^{-0.7} .
$$

2) $E c л u \mathbf{x} \in \mathscr{F}_{n}, m o$

$$
\left|E_{n}(p)\right| \stackrel{r}{\ll} n^{-\beta}, \quad \beta \geqslant \frac{8 r^{2}}{\ln r+1.5 \ln \ln r+4.2} .
$$


Далее, очевидно, что

$$
p(-t)=2 \pi \sum_{s=1}^{r}(-1)^{s} x_{s} t^{s}=2 \pi P\left(\mathbf{x}^{\prime}, t\right), \quad \mathbf{x}^{\prime}:=\left(-x_{1}, \ldots,(-1)^{r} x_{r}\right),
$$

и отметим, что вьполнены следующие соотношения, важные для оценок дискретных сумм:

$$
\sigma(\mathbf{y})=\sigma\left(\mathbf{y}^{\prime}\right), \quad \mathbf{y}=\left(y_{1}, \ldots, y_{r}\right) \in \mathbb{Z}^{r} / \mathbb{N}^{r}, \quad \mathbf{y}^{\prime}:=\left(-y_{1}, \ldots,(-1)^{r} y_{r}\right)
$$

Поэтому, следующее утверждение является следствием из леммы 2.

ЛЕмма 3. 1) Если $\mathbf{x} \in \mathscr{E}_{n}$, а $\mathbf{y}$ - рациональная точка, удовлетворяющая (23), $\widetilde{p}:=2 \pi P(\mathbf{x}-\mathbf{y}, \cdot), m o$

$$
T_{n}(p)=\sigma(\mathbf{y}) I_{n}(\widetilde{p})+\varepsilon_{n}, \quad\left|\varepsilon_{n}\right| \stackrel{r}{\ll} n^{-\alpha}, \quad \alpha=0.7 .
$$

2) $\operatorname{Ecлu~} \mathbf{x} \in \mathscr{F}_{n}$, mo (cм. maкxсе (25))

$$
\left|T_{n}(p)\right| \stackrel{r}{\ll} n^{-\beta}
$$

ЛЕмма 4 [4]. Если $n>n_{0}:=1024$, то для любого $\mathbf{x} \in \mathscr{E}_{n}$ рациональная точка $\mathbf{y}=\mathbf{a} / Q$ с условием (23) является единственной.

ДокАЗАТЕльство. В самом деле, допустим противное. Тогда найдется другая рациональная точка $\mathbf{y}^{\prime}=\mathbf{a}^{\prime} / Q^{\prime} \neq \mathbf{a} / Q$ такая, что

$$
\max \left(Q^{\prime}, \max _{1 \leqslant s \leqslant r} n^{s}\left|x_{s}-\frac{a_{s}^{\prime}}{Q^{\prime}}\right|\right) \leqslant n^{0.3} .
$$

Поскольку $\mathbf{y} \neq \mathbf{y}^{\prime}$, то найдется $s \in[1, r]$, для которого $a_{s} / Q \neq a_{s}^{\prime} / Q^{\prime}$, так что

$$
\frac{1}{Q Q^{\prime}} \leqslant\left|\frac{a_{s}}{Q}-\frac{a_{s}^{\prime}}{Q^{\prime}}\right| \leqslant 2 n^{0.3-s} \leqslant 2 n^{-0.7}, \quad \max \left(Q, Q^{\prime}\right) \leqslant n^{0.3} .
$$

Если $n>1024$, то последние две оценки противоречат друг другу. Это доказывает единственность у для $n>n_{0}$.

Зафиксируем многочлен $p=2 \pi P(\mathbf{x}, \cdot) \in \mathscr{P}^{r}$. По мере возрастания $n \geqslant n_{0}$, точка $\mathbf{x}$ поочередно пребьвает то в $\mathscr{E}_{n}$, то в $\mathscr{F}_{n}$. Соответственно этому, мы разбиваем $\mathbb{N}$ на два подмножества

$$
\mathbb{N}_{1}(p):=\left\{n \geqslant n_{0}, \mathbf{x} \in \mathscr{E}_{n}\right\}, \quad \mathbb{N}_{2}(p):=\left[1, n_{0}\right) \cup\left\{n \geqslant n_{0}, \mathbf{x} \in \mathscr{F}_{n}\right\} .
$$

Рассмотрим набор различных рациональных точек $\mathscr{Y}(\mathbf{x}):=\left\{\mathbf{y}_{1}, \mathbf{y}_{2}, \ldots\right\}$ в $\mathbb{R}^{r}$, которые последовательно являются, по мере возрастания $n$, рациональными аппроксимантами $\mathbf{y}=\mathbf{y}^{(n)}(\mathbf{x})$ вектора $\mathbf{x}$ в соответствии с (23). Таким образом, $\mathbb{N}_{1}(p)$ есть объединение непересекающихся интервалов натуральных чисел $\left[\mu_{j}, \nu_{j}\right](p)$ :

$$
\mathbb{N}_{1}(p)=\bigcup_{j \geqslant 1}\left[\mu_{j}, \nu_{j}\right](p), \quad\left[\mu_{j}, \nu_{j}\right](p):=\left\{n \geqslant n_{0}, \mathbf{y}^{(n)}(\mathbf{x})=\mathbf{y}_{j}=\frac{\mathbf{a}_{j}}{Q_{j}}\right\}
$$

Соответственно, пусть $\mathbb{N}_{2}(p)=\mathbb{N} \backslash \mathbb{N}_{1}(p)$. 
ЛЕмма 5 (см. также [4, лемма 3]). Кажддая пара последовательных интервалов $\left[\mu_{j}, \nu_{j}\right],\left[\mu_{j+1}, \nu_{j+1}\right]$ в (29) "иироко разнесена", а знаменатели $Q_{j}$ "быстро возрасmaюm":

$$
\mu_{j+1} \geqslant(0.5)^{10 / 3} \nu_{j}^{4 / 3}, \quad Q_{j+1} \geqslant 0.5 Q_{j}^{4 / 3} .
$$

ДокАЗАТЕЛЬСТво аналогично доказательству леммы 4 . Поскольку $\mathbf{y}_{j} \neq \mathbf{y}_{j+1}$, то найдется такое $s \in[1, r]$, что

$$
\frac{1}{Q_{j} Q_{j+1}} \leqslant\left|\frac{a_{s, j}}{Q_{j}}-\frac{a_{s, j+1}}{Q_{j+1}}\right| \leqslant \nu_{j}^{-0.7}+\nu_{j+1}^{-0.7} \leqslant 2 \nu_{j}^{-0.7} .
$$

Поэтому $\left(\mu_{j} \mu_{j+1}\right)^{0.3} \geqslant Q_{j} Q_{j+1} \geqslant 0.5 \nu_{j}^{0.7}$, так как $Q_{j} \leqslant \mu_{j}^{0.3}, Q_{j+1} \leqslant \mu_{j+1}^{0.3}$, что и завершает доказательство оценок (30).

Мызаключаем, что асимптотические формулы (19) и оценки (20) справедливы с $\sigma_{j}=$ $\sigma\left(\mathbf{y}_{j}\right)$ и в виду $(30),(22)$

$$
\sum_{j}\left|\sigma_{j}\right| \stackrel{r}{\ll} \sum_{j} Q_{j}^{-\rho} \stackrel{r}{\ll} 1 .
$$

Это завершает рассмотрение случая 3 ).

В отношении скорости сходимости последовательности частных сумм $S_{m}(f, p)$ в этом случае скажем, что ситуация, естественно, более сложная, чем в предыдущих случаях, см. (16) и (10). Скорость зависит от арифметических свойств вектора коэффициентов х. Для положительного числа $\varepsilon$ в $\varepsilon$-окрестности точки $\mathbf{x}$ найдем рациональный вектор $\mathbf{y} \neq \mathbf{x}$ с наименьшим знаменателем $Q(\mathbf{y})$ и обозначим этот знаменатель через $Q(p, \varepsilon)$. Ясно, что $Q(p, \varepsilon) \rightarrow \infty, \varepsilon \rightarrow 0$. Тогда из приведенной вьше конструкции вытекает, что

$$
\left|S(f, p)-S_{m}(f, p)\right| \stackrel{r}{\ll} Q^{-\rho}\left(p, m^{-0.3}\right)+m^{-\beta} .
$$

Подробности см. в [9].

Случай 4). Опять временно предположим, что последовательность $f=\left\{f_{\mathbf{n}}\right\}$ имеет компактный носитель и докажем оценку (5). Мы воспользуемся индукцией по числу элементов $a:=\# \mathscr{A}$ в $\mathscr{A}$ и главной идеей Теляковского, т.е. разбиением области суммирования на полиномиальные октанты.

Если $a=0$, т.е. $\mathscr{A}=\varnothing$, утверж дение (5) верно в силу уже рассмотренного случая 2$)$. Заметим, что если мы рассмотрим $A_{n}(p)=T_{n}(p)-T_{n-1}(p)$ как функцию коэффициентов многочлена $p$ для фиксированного $n$, то эта функция периодична по каждой из этих переменных и период $=2 \pi$. Поэтому мы можем, не теряя обшности, предположить, что коэффициенты многочлена $p_{k}$ в $A_{n_{k}}\left(p_{k}\right)$ не превосходят $\pi$ по абсолютной величине.

Согласно принципу индукции предположим

$$
\sup _{\vec{p} \in \mathscr{P}^{r, d}}|S(f, \mathscr{A}, \vec{p})| \stackrel{r, d}{\ll} 1, \quad \# \mathscr{A}=a,
$$

и выведем из этого предположения гранищу для сумм по октантам $\omega_{m}, m \in \mathbb{N}$, см. (11):

$$
S_{\omega_{m}}(f, \mathscr{A}, \vec{p}):=\sum_{\mathbf{n} \in \omega_{m}} f_{\mathbf{n}} C_{\mathbf{n}}(\mathscr{A}, \vec{p}), \quad \# \mathscr{A}=a+1 .
$$


Перепишем эти суммы, как и в [14]:

$$
S_{\omega_{m}}(f, \mathscr{A}, \vec{p})=\sum_{\mathbf{n}^{1} \in \omega_{m}^{d-1}} C_{\mathbf{n}^{1}}(\vec{p}) D_{\mathbf{n}^{1}}(f, \mathscr{A}, p)
$$

где

$$
C_{\mathbf{n}^{1}}(\mathscr{A}, \vec{p}):=\prod_{k=2}^{d} C_{n_{k}}\left(\mathscr{A}, p_{k}\right), \quad D_{\mathbf{n}^{1}}(f, \mathscr{A}, p):=\sum_{n: p_{*}(n) \geqslant p_{* 2}\left(n_{2}\right)} f_{\mathbf{n}} C_{n}(\mathscr{A}, p) .
$$

В силу (6) мы можем оценить произведение $C_{\mathbf{n}^{1}}(\mathscr{A}, \vec{p})$ тривиально, см. также (13):

$$
\left|C_{\mathbf{n}^{1}}(\mathscr{A}, \vec{p})\right| \stackrel{d}{\ll} \prod_{k=2}^{d} \frac{\min \left(1, p_{* k}\left(n_{k}\right)\right)}{n_{k}}=\Pi_{\mathbf{n}^{1}}(\vec{p}) .
$$

Что касается сумм $D_{\mathbf{n}^{1}}(f, p)$, то тут есть две возможности.

(i) $1 \notin \mathscr{A}$, так что $C_{n}(\mathscr{A}, p)=B_{n}(p)$. В этом случае мы оцениваем $D_{\mathbf{n}^{1}}(f, p)$ и $S_{\omega_{m}}(f, \mathscr{A}, \vec{p})$ с помощью $(33),(15)$, не используя предположения индукции $(31)$ :

$$
\begin{aligned}
&\left|D_{\mathbf{n}^{1}}(f, \mathscr{A}, p)\right|=\left|\sum_{n: p_{*}(n) \geqslant p_{* 2}\left(n_{2}\right)} f_{\mathbf{n}} B_{n}(p)\right| \stackrel{r}{\ll} \min \left(1, p_{* 2}-\rho\left(n_{2}\right)\right), \\
&\left|S_{\omega_{m}}(f, \mathscr{A}, \vec{p})\right| \stackrel{r, d}{\ll} \sum_{\mathbf{n}^{1} \in \omega_{m}^{d-1}} \Pi_{\mathbf{n}^{1}}(\vec{p}) \min \left(1, p_{* 2}-\rho\left(n_{2}\right)\right) \stackrel{r, d}{\ll} \min \left(1, p_{*}-\rho(m)\right) .
\end{aligned}
$$

(ii) $1 \in \mathscr{A}$, так что $C_{n}(\mathscr{A}, p)=A_{n}(p)$ и

$$
D_{\mathbf{n}^{1}}(f, \mathscr{A}, p)=\sum_{n: p_{*}(n) \geqslant p_{*_{2}}\left(n_{2}\right)} f_{\mathbf{n}} A_{n}(p)=\sum_{n: p_{*}(n) \geqslant p_{*_{2}}\left(n_{2}\right)} f_{\mathbf{n}}\left(T_{n}(p)-T_{n-1}(p)\right) .
$$

Здесь нам потребуется воспользоваться предположением, что коэффициенты многочлена $p$ не превосходят $\pi$ по абсолютной величине. Соответственно, из условия $p_{*}(n) \geqslant$ $p_{* 2}\left(n_{2}\right)$ вытекает

$$
n \gg p_{* 2}^{\rho}\left(n_{2}\right), \quad n^{-\beta} \ll \min \left(1, p_{* 2}^{-\gamma}\left(n_{2}\right)\right), \quad \gamma:=\rho \beta .
$$

Рассмотрим числовую последовательность $\left\{\varepsilon_{n}\right\}$, удовлетворяющую оценке $\left|\varepsilon_{n}\right| \ll n^{-\beta}$, а также последовательность $f=\left\{f_{n}\right\} \in \mathscr{S},\|f\|_{\mathscr{S}} \leqslant 1$. Применяя преобразование Абеля (см. также (8)), мы видим, что

$$
\left|\sum_{n \geqslant m} f_{n}\left(\varepsilon_{n}-\varepsilon_{n-1}\right)\right|=\left|\varepsilon_{m-1} f_{m}-\sum_{n \geqslant m} \varepsilon_{n} \Delta f_{n}\right| \stackrel{\beta}{\ll} m^{-\beta},
$$

и поэтому из (36) следует, что

$$
\left|\sum_{n: p_{*}(n) \geqslant p_{* 2}\left(n_{2}\right)} f_{\mathbf{n}}\left(\varepsilon_{n}-\varepsilon_{n-1}\right)\right| \ll \min \left(1, p_{* 2}{ }^{-\gamma}\left(n_{2}\right)\right)
$$


Отсюда, рассуждая в точности так же, как и вьше, мы получаем

$$
\begin{aligned}
& \left|\sum_{\mathbf{n}^{1} \in \omega_{m}^{d-1}} C_{\mathbf{n}^{1}}(\mathscr{A}, \vec{p})\left(\sum_{n: p_{*}(n) \geqslant p_{* 2}\left(n_{2}\right)} f_{\mathbf{n}}\left(\varepsilon_{n}-\varepsilon_{n-1}\right)\right)\right| \\
& \stackrel{r, d}{\ll} \sum_{\mathbf{n}^{1} \in \omega_{m}^{d-1}} \Pi_{\mathbf{n}^{1}}(\vec{p}) \min \left(1, p_{* 2}^{-\gamma}\left(n_{2}\right)\right) \stackrel{r, d}{\ll} \min \left(1, p_{*} \bar{d}^{-\gamma}(m)\right) .
\end{aligned}
$$

С помощью этой оценки мы "вьметаем" суммирование остаточных членов $\varepsilon_{n}$ асимптотических формул (26), а также суммирование по $n \in \mathbb{N}_{2}(p)$. С точностью до равномерно ограниченной ошибки суммирование по $n$ локализуется на главные члены асимптотических формул на множестве $\mathbb{N}_{1}(p)=\bigcup_{j}\left[\mu_{j}, \nu_{j}\right](p)$. Из $(26),(27)$ мы видим, что

$$
\left|S_{\omega_{m}}(f, \mathscr{A}, \vec{p})-\sum_{j} \sigma_{j} S\left(f^{(j, m)}, \vec{p}_{j}^{\prime}\right)\right| \stackrel{r, d}{\ll} \min \left(1, p_{*}{ }^{-\gamma}(m)\right),
$$

где

$$
\begin{gathered}
\vec{p}_{j}^{\prime}=\left(\widetilde{p}_{j}, p_{2}, \ldots, p_{d}\right), \quad S\left(f^{(j, m)}, \vec{p}_{j}^{\prime}\right)=\sum_{\mathbf{n} \in \mathbb{N}^{d}} f_{\mathbf{n}}^{(j, m)} B_{n}\left(\widetilde{p}_{j}\right) C_{\mathbf{n}^{1}}(\vec{p}), \\
f_{\mathbf{n}}^{(j, m)}:= \begin{cases}f_{\mathbf{n}} & \text { для } \mathbf{n} \in \omega_{m}, n \in\left[\mu_{j}, \nu_{j}\right](p), \\
0 & \text { для всех остальных } \mathbf{n} \in \mathbb{N}^{d} .\end{cases}
\end{gathered}
$$

Для любой фиксированной пары $m, j \in \mathbb{N}$ множество $\left\{\mathbf{n}: \mathbf{n} \in \omega_{m}, n \in\left[\mu_{j}, \nu_{j}\right](p)\right\}$ координатно вьпуклое (возможно пустое), так что последовательность $f^{(j, m)}:=$ $\left\{f_{\mathbf{n}}^{(j, m)}\right\}_{\mathbf{n} \in \mathbb{N}^{d}}$ координатно медленная и при этом $\left\|f^{(j, m)}\right\|_{\mathscr{S}^{d}} \leqslant 2\|f\|_{\mathscr{S}^{d}} \leqslant 2$. Далее, общее число сомножителей типа $A$ в произведении $B \prod_{k=2}^{d} C$ на единицу менњше, чем в исходном произведении $\prod_{k=1}^{d} C$. Поэтому, пользуясь предположением индукции (31), мы видим, что

$$
\left|S\left(f^{(j, m)}, \vec{p}_{j}^{\prime}\right)\right| \stackrel{r, d}{\ll} 1 .
$$

Поскольку также $\sum_{j}\left|\sigma_{j}\right| \stackrel{r}{\ll} 1$, то из (37) следует, что

$$
\left|S_{\omega_{m}}(f, \mathscr{A}, \vec{p})\right| \stackrel{r, d}{\ll} 1, \quad \# \mathscr{A}=a+1 .
$$

Отсюда вытекает глобальная ограниченность всей суммы для $f$ с компактньп носителем:

$$
\sup _{\vec{p} \in \mathscr{P}^{r, d}}|S(f, \mathscr{A}, \vec{p})|<\infty
$$

В завершение докажем сходимость ряда $S(f, \mathscr{A}, \vec{p})$ для медленных последовательностей $f$, не имеющих компактного носителя. С этой целью достаточно установить, что для любого фиксированного набора многочленов $\vec{p} \in \mathscr{P}^{r, d}$

$$
\sup _{f \in \mathscr{S}_{0}^{d}}\left|S_{\omega_{m}}(f, \mathscr{A}, \vec{p})\right| \rightarrow 0, \quad m \rightarrow \infty
$$


где $\mathscr{S}_{0}^{d}$ обозначает множество всех медленных последовательностей с компактным носителем. Мы можем считать, что ни один из многочленов $p_{k}$ не равен нулю тождественно, так что правые части (34) (случай (i)) и (37) (случай (ii)) равномерно малы на $\mathscr{S}_{0}^{d}$. В частности, мы можем ограничиться рассмотрением случая (ii).

Предположим, что одна из координат вектора коэффициентов $\mathbf{x}$ многочлена $P(\mathbf{x}, \cdot)=$ $p(\cdot) /(2 \pi)$ есть иррациональное число. Тогда для любого фиксированного $j$ множество $\left\{\mathbf{n} \in \omega_{m}, n \in\left[\mu_{j}, \nu_{j}\right](p)\right\}$ пустое для всех достаточно больших $m$, так что

$$
f^{(j, m)} \equiv 0, \quad m \geqslant M(j)
$$

В самом деле, из условия $\mathbf{n}=\left(n, n_{2}, \ldots, n_{d}\right) \in \omega_{m}$ вытекает, по определению (11) октанта $\omega_{m}$, что $p_{*}(n) \geqslant p_{* d}(m)$. Это противоречит границе $n \leqslant \nu_{j}(p)$, если $m$ достаточно большое.

Таким образом, существует последовательность $\{J(m)\}_{m \in \mathbb{N}}$ такая, что

$$
J(m) \rightarrow \infty, \quad m \rightarrow \infty, \quad S\left(f^{(j, m)}, \vec{p}_{j}^{\prime}\right)=0, \quad j \leqslant J(m),
$$

и следовательно, в силу (37)

$$
S_{\omega_{m}}(f, \mathscr{A}, \vec{p}) \stackrel{r, d}{\ll} p_{*}{ }^{-\gamma}(m)+\left(\sum_{j>J(m)} \sigma_{j}\right) \rightarrow 0, \quad m \rightarrow \infty .
$$

Наконец, если $\mathbf{x} \in \mathbb{Z}^{r} / \mathbb{N}$, то множество $\mathbb{N}_{1}(p)=\bigcup_{j}\left[\mu_{j}, \nu_{j}\right]$ завершается полуосью $\left[\mu_{J}, \infty\right)$ и $\widetilde{p}_{J} \equiv 0$, так что для $n \geqslant \mu_{J}$ мы попросту имеем $B_{n}\left(\widetilde{p}_{J}\right) \equiv 0$. Следовательно, если $m$ достаточно велико, то $S\left(f^{(j, m)}, \vec{p}_{j}^{\prime}\right)=0$ для всех $j$, и для всех таких $m$

$$
S_{\omega_{m}}(f, \mathscr{A}, \vec{p}) \stackrel{r, d}{\ll} p_{*}-\gamma(m),
$$

что и завершает доказательство леммы 1.

\section{СПИСОК ЦИТИРОВАННОЙ ЛИТЕРАТУРЫ}

[1] Теляковский С. А. Об оценках производных тригонометрических полиномов многих переменных // Сиб. матем. ж. 1963. Т. 4. №6. С. 1404-1411.

[2] Теляковский С. А. Равномерная ограниченность некоторых тригонометрических полиномов многих переменных // Матем. заметки. 1987. Т. 42. №1. С. 33-39.

[3] Теляковский С. А., Темляков В.Н. О сходимости рядов Фурье многих переменных ограниченной вариации // Матем. заметки. 1997. Т. 61. № 4. С. 583-591.

[4] Архипов Г.И., Осколков К.И. Об одном специальном тригонометрическом ряде и его применениях // Матем. сб. 1987. Т. 134 (176). № 2 (10). С. 147-157.

[5] Виноградов И. М. Метод тригонометрических сумм в теории чисел. М.: Наука, 1971.

[6] Stein E., Wainger S. Discrete analogues of singular Radon transforms // Bull. Amer. Math. Soc. 1990. V. 23. P. 537-544.

[7] Oskolkov K. I. A class of I.M. Vinogradov's series and its applications in harmonic analysis // Progress in Approximation Theory. An International Prospective Proceedings of the International Conference on Approximation Theory held March 19-22, 1990, at the University of South Florida: Springer-Verlag, 1992. P. 353-402.

[8] Oskolkov K. I. Schrödinger equation and oscillatory Hilbert transforms of second degree // J. Fourier Anal. Appl. 1998. V. 4. P. 341-356. 
[9] Осколков К.И. Ряды И.М. Виноградова в задаче Коши для уравнения Шрёдингера // Тр. МИАН. 1991. Т. 200. С. 265-288.

[10] Zygmund A. Trigonometric series. Second Edition. Cambridge: Cambridge University Press, 1959.

[11] Garaev M.Z. On a multiple trigonometric series // Acta Arithm. 2002. V. 102. № 2. P. 183-187.

[12] Stein E., Wainger S. The estimation of an integral arising in multiplier transformations // Studia Math. 1970. V. 35. P. 101-104.

[13] Stein E. Harmonic Analysis: Real-Variable Methods, Orthogonality and Oscillatory Integrals. Princeton, NJ: Princeton University Press, 1993.

[14] Weyl H. Über die Gleichverteilung von Zahlen mod Eins // Math. Ann. 1916. V. 77. P. 313-352.

[15] Jing-run Chen. On Professor Hua's estimate of exponential sum. // Sci. Sinica. 1977. V. 20. P. 711-719.

[16] Стечкин С. Б. Оценка полной рациональной тригонометрической суммы // Тр. МИАН. 1977. T. 143. C. $188-207$.

[17] Архипов Г. И. О проблеме Гильберта-Камке // Изв. АН СССР. Сер. матем. 1984. Т. 48. № 1 . C. $3-52$.

Университет Южной Каролины,

Поступило

г. Колумбия, США

29.01.2003 